\title{
Automated neurosurgical video segmentation and retrieval system
}

\author{
Engin Mendi ${ }^{1}$, Songul Cecen ${ }^{2}$, Emre Ermisoglu $^{2}$, Coskun Bayrak $^{2}$ \\ ${ }^{1}$ Department of Applied Science, University of Arkansas at Little Rock, Little Rock, USA; \\ ${ }^{2}$ Department of Computer Science, University of Arkansas at Little Rock, Little Rock, USA. \\ Email: esmendi@ualr.edu; sxcecen@ualr.edu; exermisoglu@ualr.edu; cxbayrak@ualr.edu
}

Received 18 January 2010; revised 25 February 2010; accepted 6 March 2010.

\begin{abstract}
Medical video repositories play important roles for many health-related issues such as medical imaging, medical research and education, medical diagnostics and training of medical professionals. Due to the increasing availability of the digital video data, indexing, annotating and the retrieval of the information are crucial. Since performing these processes are both computationally expensive and time consuming, automated systems are needed. In this paper, we present a medical video segmentation and retrieval research initiative. We describe the key components of the system including video segmentation engine, image retrieval engine and image quality assessment module. The aim of this research is to provide an online tool for indexing, browsing and retrieving the neurosurgical videotapes. This tool will allow people to retrieve the specific information in a long video tape they are interested in instead of looking through the entire content.
\end{abstract}

Keywords: Video Processing; Video Summarization; Video Segmentation; Image Retrieval; Image Quality Assessment

\section{INTRODUCTION}

Developing countries suffer from lack of access to the medical expertise. Due to the inadequacy of trained medical professionals, the health maintenance system of the country may face variety of problems which will directly affect individual's quality of life and also entire wellbeing of society. Limitations in accessing to the medical expertise may also exist in small regional hospitals \& health-care centers in rural places in developed countries. Therefore, connecting as many hospitals as possible to a medical information system from regional level to the state and national levels and ultimately to the global level, which is simply illustrated in Figure 1, would be very beneficial in terms of improved standard of medical practice and educational aspects for medical students and staff who can not reach to the medical resources, due to resource, geographical, and time constraints.

Our research is to show not only the importance of the accommodation of the massive amount of data for educational use but also the preservation of a life long experience of pioneers in the field of neurosurgery for further use via automatically defining a logical structure of the video content. Since only a few fortunate ones get a chance to be with these experts to see how they perform such complex operations. Therefore, with this service the boundaries can be expended so that [1].

- The educational needs of residents can be complemented by allowing access in a timely efficient manner.

- The educational needs of medical students can be provided by allowing access.

- The knowledge enhancement needs of Neurosurgeons around the world with special benefits to developing countries can be supported by allowing access.

- The help in teaching of operating room (OR) nurses and physician assistants can be provided.

- The foundation for future research related to simulation technology can be constructed.

The goal of this system is summarized in Figure 2. The system has 3 main components which are video segmentation engine, image retrieval engine and image quality assessment module.

\section{VIDEO SEGMENTATION ENGINE}

Medical video libraries are dedicated to many healthrelated applications such as medical imaging, medical research and education, medical diagnostics and training of medical professionals. Due to the rapid development in production, storage and distribution of multimedia content, the video data of these medical repositories can be directly transmitted to the people via internet. However, due to the huge size of the videos, very large bandwidth will be required. Additionally, it will be very difficult reaching a certain portion of the video. For instance, when a medical surgeon or student wants to look through a spe- 


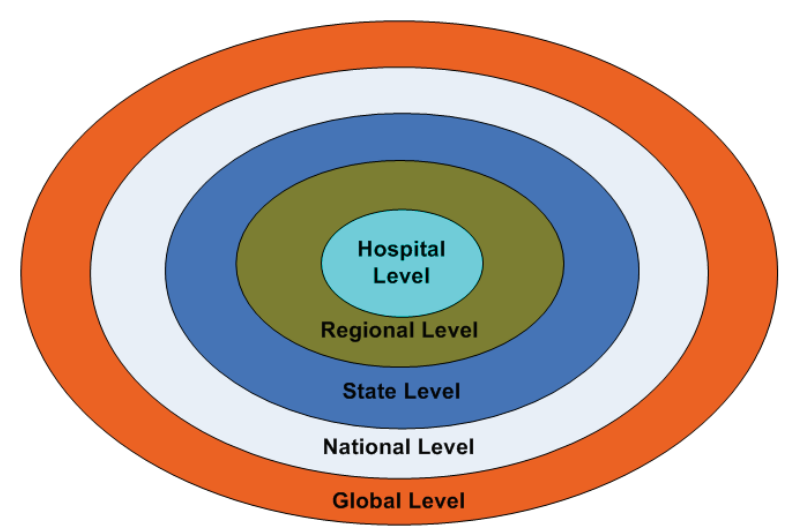

Figure 1. Work-flow of the system.

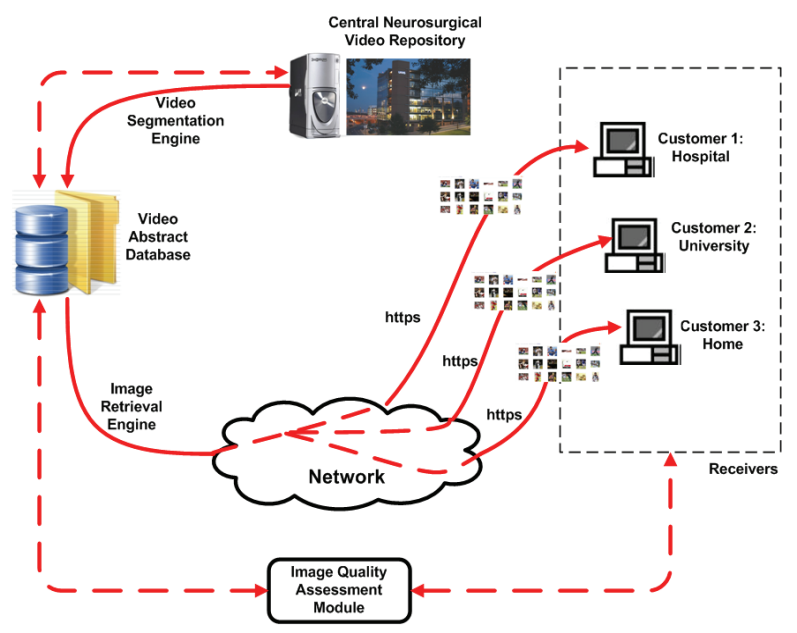

Figure 2. System architecture.

cific part of a 15 -hour neurosurgery videotape, they will have to browse the entire content of the video in order to find the right part they want to see. Our video segmentation engine generates a concise summary of the semantics in the neurosurgical videotape to help them browse and search the large amount of video data. The architecture of the engine is depicted in Figure 3. As shown in Figure 3, an MPEG video source comprises a group of video shots, and a video shot is an unbroken sequence of frames captured from one perspective. The engine partitions a video sequence into a set of shots, and some key frames are extracted to represent each shot. Finally key frames are collected in the video abstract database.

Video segmentation is the central process for automatic video indexing, browsing and retrieval systems. It aims partitioning a video sequence into meaningful segments and extracting a sequence of key frames, that each key represents the content of corresponding video segment. The video sequence is divided into meaningful segments (shots) which are the basic elements of index- ing, and then each shot is represented by key frames. These frames are indexed by extracting spatial and temporal features.

Video segmentation includes two major steps: 1) Shot boundary detection and 2) Key frame extraction. Shot boundary detection targets breaking up the video into meaningful sub-segments. Key frame extraction involves selecting one or multiple frames that will represent the content of each shot.

In our work, we segmented the shots by detecting the boundaries via color histogram differences and selfsimilarity analysis. In color histogram differences, RGB color space is converted to HSV space, and then colorquantization is applied to HSV color space. Finally the differences of HSV histograms between consecutive frames are computed to determine the peaks representing the shot boundaries $[1,2]$. In self-similarity analysis, HSV feature vectors of the frames within the video data are visualized with a two-dimensional matrix by applying a similarity metric [2].

Key frame extraction is the second major process of video segmentation. We used four approaches in order to select the key frames:

1) The first is the traditional k-means clustering, determining video summaries with a specific number of frames, which will represent the entire video content. The number of the key frames is specified by the user. The frames closest to the cluster centroids are selected as key frames [2].

2) The second is the dominant set clustering that automatically decide the number of key frames according to the similarity of the data without any initial decision of cluster number. The clustering is based on dominant sets which are the representation of an edge-weighted graph as a similarity matrix [2-4].

3) The third is based on salient region detection and structural similarity. Saliency maps representing the attended regions are produced from the color and luminance features of the video frames. Introducing a novel signal fidelity measurement-saliency based structural similarity index, the similarity of the maps is measured.

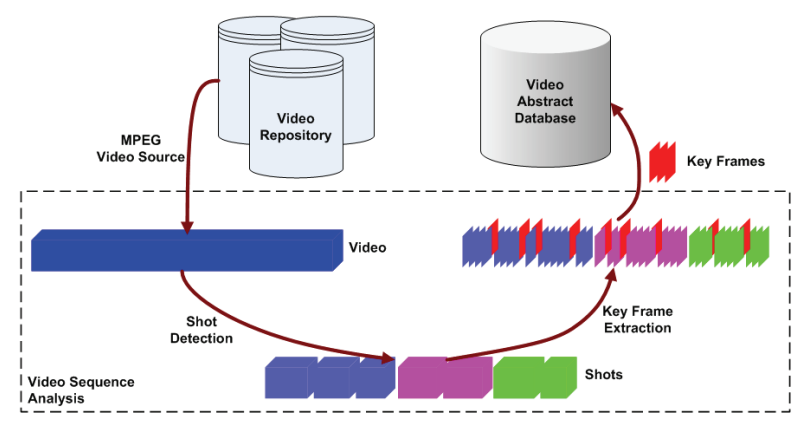

Figure 3. The architecture of the video segmentation engine. 
Based on the similarities, shot boundaries and key frames are determined [5].

4) The fourth approach, called "index-based retrieval (i-Base)", is based on Discrete Cosine Transform (DCT) and Self Organizing Map (SOM). It allows user to quickly find a particular point within a certain domain and/or determine if the domain is relevant to the need. i-Base forms a hierarchy from the uniquely represented shots by using frames. User then can map only the relevant section in the source with the request issued. The idea of 'just request-to-response mapping' prevents not only the unwanted information retrieval but also saves time and bandwidth [1].

Figure 4 shows the user interfaces of the video segmentation system, allowing the user to set the parameters. Currently, we are in the process of transferring our tool over the WEB environment. A sample of key frame set of a neurosurgical training video data, presented to the user is depicted in Figure 5.

\section{IMAGE RETRIEVAL ENGINE}

Currently, all web-browser based image search engines are based on textual data, that images are associated by annotations and then searched using keywords. In terms of medical images, the most of the medical image information is not accessible or limited to one or two databases that can be searched with key words. As medical images can not be fully described by textual information, keywords are not sufficient enough to retrieve relevant medical images from large databases. For instance, for the "lung CT" key term, Google is able to retrieve only 130 image results. However, only Health Education As sets Library (HEAL, http://www.healcentral.org) contains
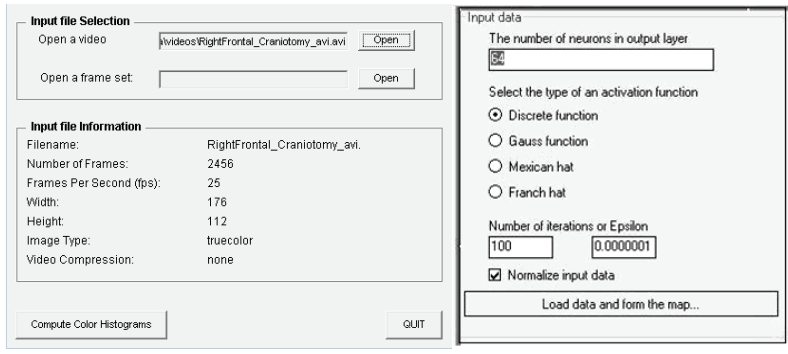

Figure 4. User interface of the tool.

more than 1,000 lung CT images [6].

Our image retrieval engine delivers the image results from our video abstract database, taking advantage of visual features of the images. Figure 6 shows the architecture of our image retrieval engine. Several image features representing the visual content of the images in video abstract database and query image are extracted. The images in video abstract database are the key frames of the neurosurgical videotapes previously extracted by the video segmentation engine.

Based on the similarity metric, how close query image and key frames are measured. Retrieval results are then ranked according to the similarity score and delivered to make available to receivers over broadband network.

Content-based image retrieval (CBIR) is a technique using visual descriptors to search images from image databases according to users' needs. It aims effectively searching and browsing of large image digital libraries based on automatically extracted image features. In a typical CBIR system, features of every image in the database have been extracted and then compared with the query image. We have conducted a preliminary evalu-

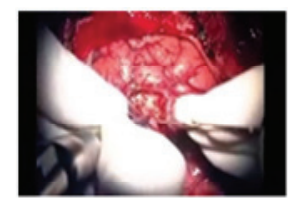

Frame 174

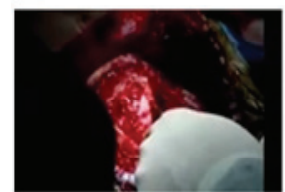

Frame 680

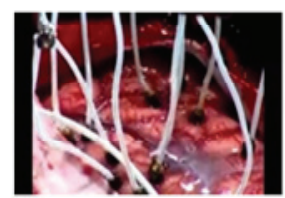

Frame 1324

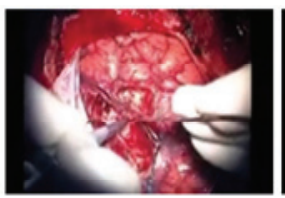

Frame 224

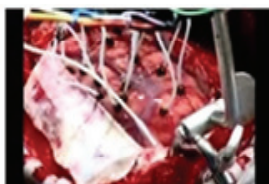

Frame 724

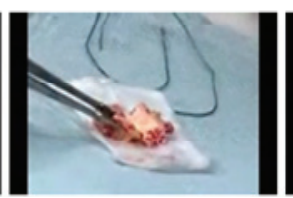

Frame 1342

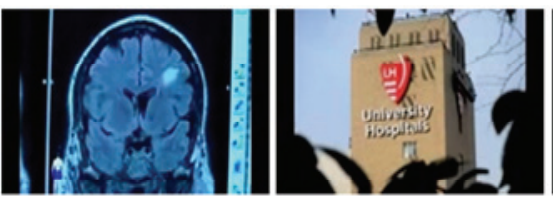

Frame 324

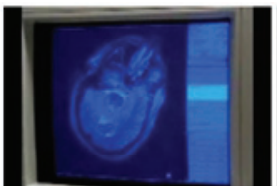

Frame 899

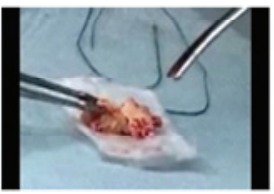

Frame 1374
Frame 474

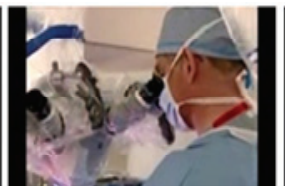

Frame 974

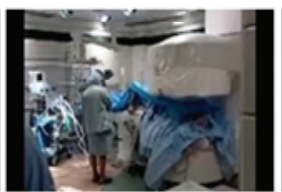

Frame 1524

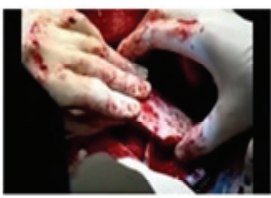

Frame 599

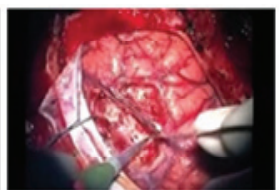

Frame 1049

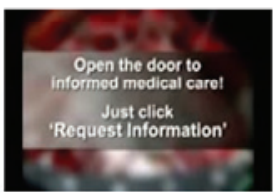

Frame 1649

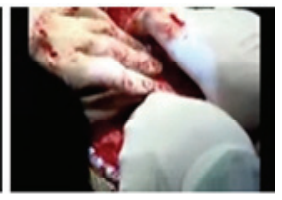

Frame 654

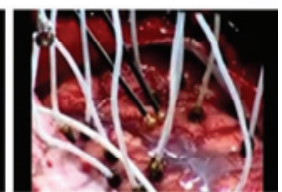

Frame 1174

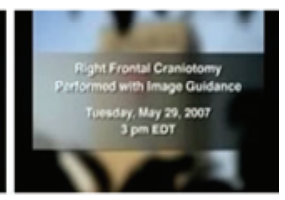

Frame 1974

Figure 5. The 18 key frames of a neurosurgical (right frontal craniotomy) video sequence of 2500 frames presented to the user. 


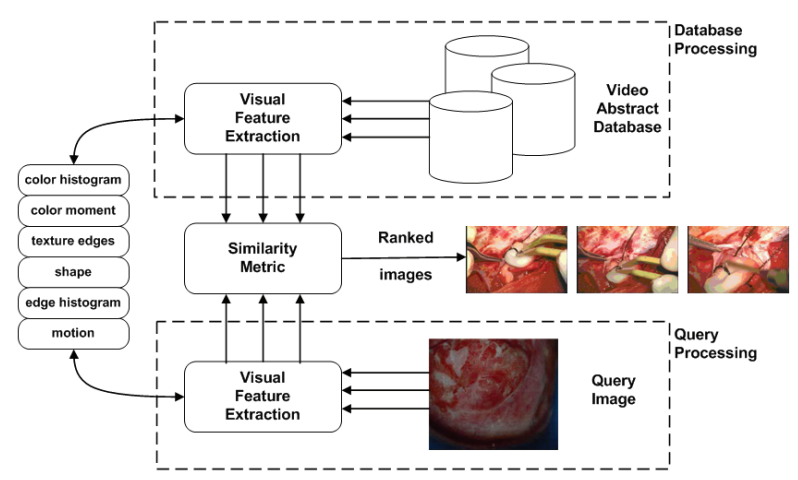

Figure 6. The architecture of the image retrieval engine.

ation on the precision performance of following two approaches:

1) The first is comparing images using color histograms. Color is one of the most important image features for CBIR. A color histogram is the representation of frequency distribution of color bins in an image. Color histograms are widely used in comparison of images, since they are robust to change in translation, rotation and angle of view. We have used two different color spaces, RGB and HSV. We quantized the RGB color space as well as HSV space to reduce the number of bins, using 256 colors (16 levels for each R, G and B channels in RGB space; 16 levels for $\mathrm{H}$ channel, 4 levels for $\mathrm{S}$ channel and 4 levels for V channel in HSV space). Finally, to evaluate the similarity between query image and the image in the video abstract database, we have computed the Euclidean distance between corresponding color histograms [7].

2) The second is comparing images using two image fidelity measurements. We have used mean squared error (MSE) and structural similarity (SSIM) index [8] to quantify the similarity of images [7]. MSE compares two images on a pixel-by-pixel basis, whereas SSIM considers structural information.

We have conducted a preliminary evaluation on the precision performance of above four approaches. We have used a subset of COREL Image Database $[9,10]$ which is available at http://wang.ist.psu.edu/docs/related. shtml. The database contains 10 image classes with 100 images each (1000 images in total). The classes are: Africa, Beach, Buildings, Buses, Dinosaurs, Flowers, Elephants, Horses, Food and Mountains.

We measured the retrieval effectiveness on the precision performance of each approach. The detailed precision results are shown in Table 1. Average precisions are computed by taking every image in a class as query image. As can be seen, the precision performances of the algorithms change with different classes. According to the overall results, HSV histogram is the most effective approach among others.

\section{IMAGE QUALITY ASSESSMENT MODULE}

Image quality assessment is an important part of content delivery over networks since network conditions vary for individual users and also digital images are subject to a wide variety of distortions during processing, storage and transmission, any of which may result in a degradation of visual quality $[8,11]$. Therefore quantifying the image quality degradation occurring in a system would be very beneficial, so that the quality of the images and videos produced can be controlled and adjusted. For instance, a system can examine the quality of videos and images being transmitted in order to control and allocate streaming or downloading resources. Moreover, a quality assessment module can assist in the optimal design of pre-filtering and bit assignment algorithms at the encoder and of optimal reconstruction, error concealment, and post-filtering algorithms at the decoder [8].

On the other hand, according to a recent research of Microsoft [12]; due to the difficulty of the image quality assessment problem, current web-browser based image search engines lack of user requirements, because there is no effective and practical solution to allow an understanding of image content, which fits the user needs. Image quality assessment research would greatly help improve users' browsing experiences.

Therefore we have been designing a quality assessment module automatically predicting perceived image quality. This problem is more competitive in medical imagery, because medical imagery may play crucial role in many health-related issues such as diagnostic design, patient-care, training and education of medical professionals and students. The framework of the module is depicted in Figure 7. We have already developed a novel objective image quality metric which is superior to the existing metrics in the literature. We have also validated our metric against a large set of subjective ratings gathered

Table 1. Precision of 10 image categories for top 30 matches.

\begin{tabular}{ccccc}
\hline & \multicolumn{2}{c}{ Histogram-based } & \multicolumn{2}{c}{ Similarity-based } \\
\hline Image Class & $R G B$ & HSV & SSIM & $M S E$ \\
\hline African People & 23.03 & 40.57 & 8.33 & 0.33 \\
Beach & 13.4 & 22.63 & 33 & 33.33 \\
Buildings & 18.6 & 24.73 & 14.33 & 3.67 \\
Buses & 31 & 47.03 & 25.33 & 3.67 \\
Dinosaurs & 34.83 & 44.6 & 95.33 & 97.67 \\
Elephants & 18.8 & 19.57 & 25.33 & 35 \\
Flowers & 33.3 & 32.7 & 67 & 90 \\
Horses & 16.83 & 81.93 & 21.33 & 43.67 \\
Mountains and & 13.9 & 38 & 19 & 31 \\
glaciers & 55.03 & 31.3 & 10.33 & 1.67 \\
Food & 25.87 & 38.31 & 31.93 & 34.00 \\
AVERAGE & & & &
\end{tabular}




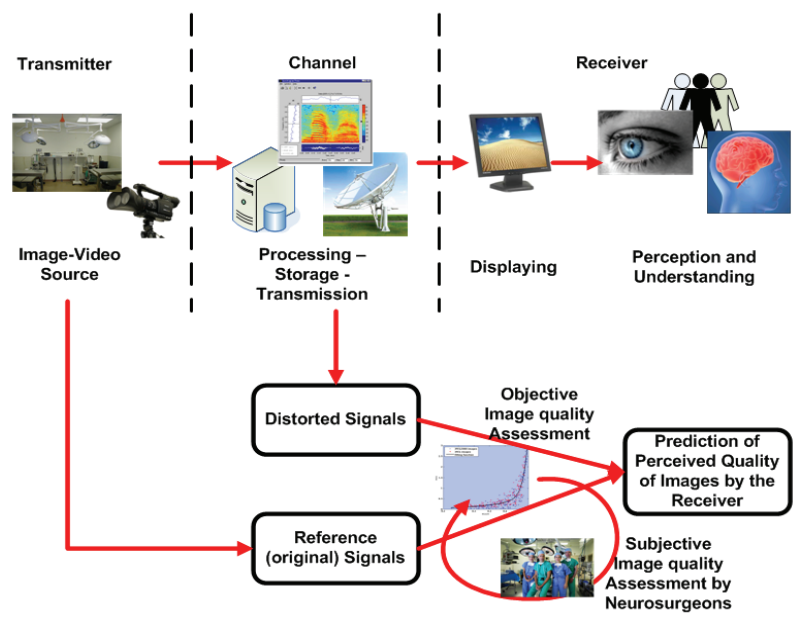

Figure 7. The framework of the image quality assessment module.

for a public image database. Currently we have been working on a subjective image quality assessment for neurosurgery imagery. This assessment will be based on expert opinions of a group of neurosurgeons from UAMS,

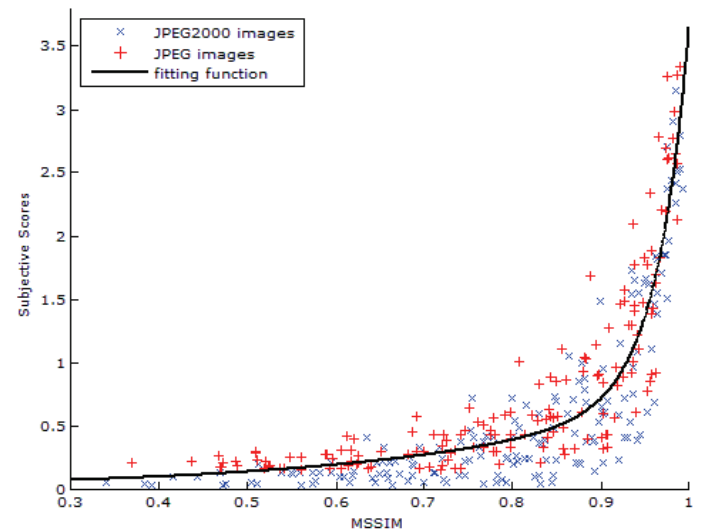

(a)

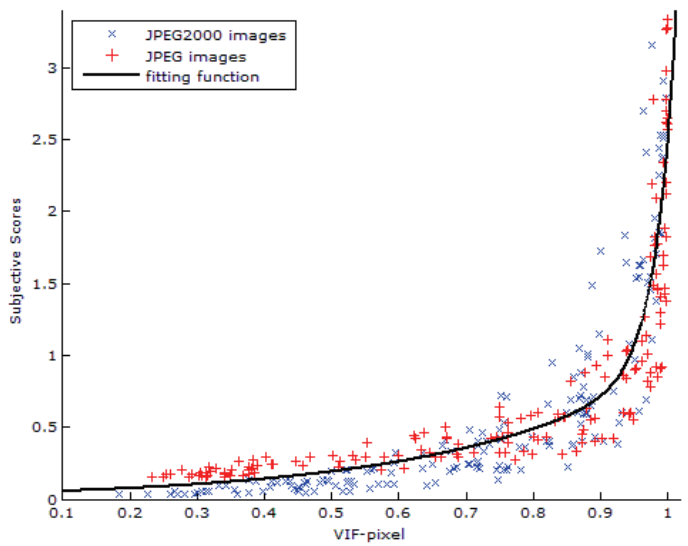

(c) by determining fixation points on the images while tracking their eye movements.

Image quality assessment has a great importance in several image and video processing applications such as filter design, image compression, restoration, denoising, reconstruction, and classification. The goal of image quality assessment is predicting image quality of display output perceived by the end user. Multimedia contents are subjected to the variety of artifacts during acquisition, processing, storage and delivering, which may lead to reductions in the quality. Our image quality assessment module dynamically monitor and adjust the image quality, so that the output quality of the image or video presented to the user can be maximized for available resources such as network conditions and bandwidth requirements.

Image quality metrics can be classified into 2 categories: Subjective and objective metrics. The most reliable way to measure of image quality is to look at it because human eyes are the ultimate viewer and images are evaluated by humans. Subjective evaluation by orienting on human visual system is determined by Mean Opinion Score (MOS) which relies on human perception. On the other hand, objective metrics are also very valuable to

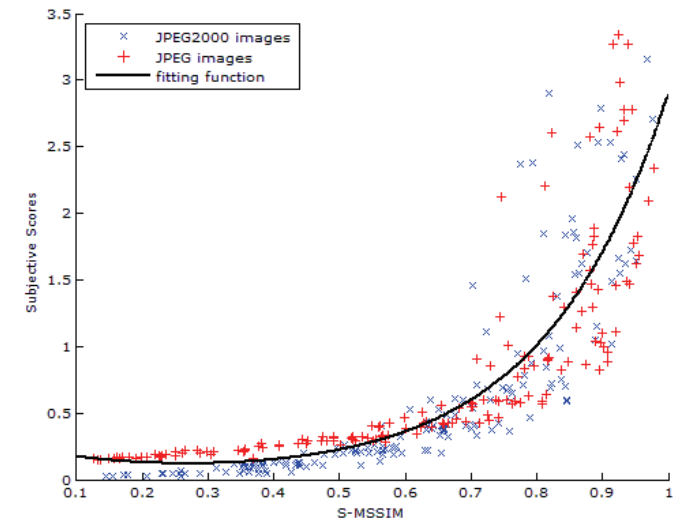

(b)

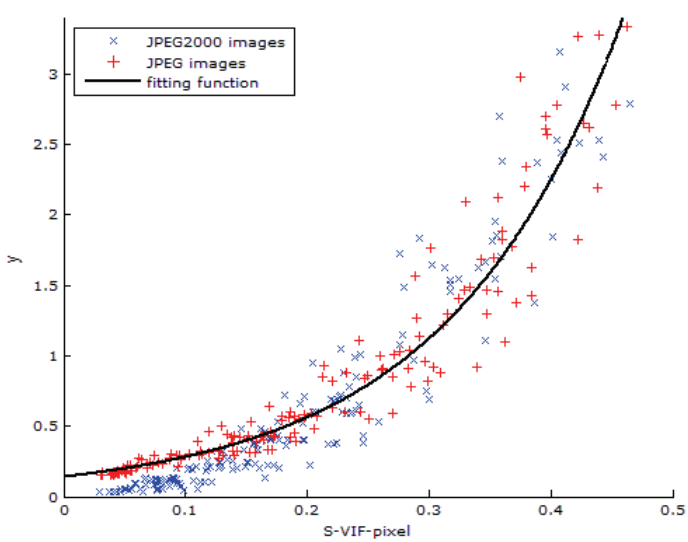

(d)

Figure 8. Scatter plots of subjective/objective scores on LIVE Database. Red points $(+)$ and blue points (x) denote JPEG and JPEG2000 images, respectively. (a) SSIM; (b) S-SSIM; (c) VIF in pixel domain; (d) S-VIF in pixel domain. 
predict perceived image quality. They are based on mathematical models that approximate results of subjective quality assessment. Amongst the objective quality metrics, full reference metrics require complete availability of original non-distorted reference image which will be compared with the corresponding distorted image, while reduced reference and no reference metrics require limited and no availability of this, respectively.

We developed a new image quality metrics, S-SSIM (saliency-based structural similarity index) and S-VIF (saliency-based visual information fidelity), based on frequency-tuned salient region detection introduced by [13]. Saliency maps are produced from the color and luminance features of the image. SSIM [8] index and visual information fidelity (VIF) in pixel domain [14] are modified by the weighting factors of the saliency maps.

We validated our approach using LIVE Image Database [15] as test bed. The database contains 29 original images and 460 distorted images (227 JPEG2000 images and 233 JPEG images) with subjective scores for each image. Non-linear regression analysis has been performed to fit the data. The Pearson correlation coefficient is used to measure the association between subjective and objective scores. Our results showed that our technique is more correlated with human subjective perception.

Figure 8 shows the results for the database. Each sample point represents the subjective/objective scores of one test image. The y axis in the figure denotes the subjective scores in the database. The $\mathrm{x}$ axis denotes the predicted quality of images after a nonlinear regression toward above 4 objective scores, which are SSIM, SSSIM, VIF in pixel domain and S-VIF in pixel domain, respectively. The Pearson validation scores between assessment metrics are depicted in Table 2 [16].

The Pearson correlation coefficient varying from -1 to 1 is widely used to measure the association between two variables. High absolute values mean that the two variables being evaluated have high correlation. As shown in Table 2, our metric is more correlated with human subjective perception.

\section{CONCLUSIONS}

We presented a medical video segmentation and retrieval research initiative. We introduced the key components of the framework including video segmentation engine, image retrieval engine and image quality assessment module. We are currently in the process of transferring our frame

Table 2. Pearson correlation coefficients.

\begin{tabular}{ccccc}
\hline & SSIM & S-SSIM & VIF-pixel & S-VIF-pixel \\
$\begin{array}{c}\text { LIVE Image } \\
\text { Database }\end{array}$ & 0.6823 & 0.7475 & 0.7126 & 0.9083 \\
\hline
\end{tabular}

work and software tool over the WEB environment. This will allow people to access the specific information that they are interested in among entire video. Multimedia information system, digital library, and movie industry are some of the applications work on videos. Since they are widely used, it brings out the need of processing and saving the digital video. These processes are mainly the compressing, segmenting, and indexing of the video. The neurosurgical data which is initially compressed will pass through the segmentation and indexing. Then receiver will be able to retrieve the specific section of the video that he/she is interested in with maximum quality for the available network, bandwidth and hardware resources. The overall objective is to provide convenience and easiness in accessing the relevant data without going over the whole data.

\section{REFERENCES}

[1] Cecen, S. (2009) Histogram based video segmentation and key frame extraction on SOM and DFT. Master's Thesis, University of Arkansas, Little Rock.

[2] Mendi, E. and Bayrak, C. (2010) Shot boundary detection and key frame extraction from video sequences. Elsevier Information Sciences, 2010.

[3] Pavan, M. and Pelillo, M. (2003) Dominant sets and hiera-rchical clustering. Proceedings of the 9th European Conference on Computer Vision, 362-369.

[4] Pavan, M. and Pelillo, M. (2005) Efficient out-of-sample extension of dominant-set clusters. Advances in Neural Information Processing Systems, 17, 1057-1064.

[5] Mendi, E. and Bayrak, C. (2010) Shot boundary detection and key frame extraction using salient region detection and structural similarity. The 48th ACM Southeast Conference, Oxford, Mississippi, 15-17 April, 2010.

[6] Lehmann, T. M., Mller, H., Tian, Q., Galatsanos, N.P. and Mlynek, D. (2005) Augmented medical image management for integrated healthcare solutions.

[7] Mendi, E. and Bayrak, C. (2010) Performance analysis of color image retrieval. The 3rd International Congress on Image and Signal Processing (CISP'10), Yantai, 2010.

[8] Wang, Z. Bovik, A.C. Sheikh, H.R. and Simoncelli, E.P. (2004) Image quality assessment: From error visibility to structural similarity. IEEE Transactions on Image Processing, 13(4), 600-612.

[9] Li J. and Wang, J.Z. (2003) Automatic linguistic indexing of pictures by a statistical modeling approach IEEE Transactions on Pattern Analysis and Machine Intelligence, 25(9), 1075-1088.

[10] Wang J.Z., Li, J. and Wiederhold G. (2001) SIMPLIcity: Semantics-sensitive integrated matching for picture libraries. The IEEE Transactions on Pattern Analysis and Machine Intelligence, 23(9), 947-963.

[11] Chono, K., Lin, Y.-C., Varodayan, D., Miyamoto, Y. and Girod, B. (2008) Reduced-reference image quality assessment using distributed source coding. IEEE International Conference on Multimedia and Expo, 2008. 
[12] Zhang, L., Chen, L., Jing, F. and Ma, W.-Y. (2006) Enjoy photo a vertical image search engine for enjoying high-quality photos. The 14th ACM International Conference on Multimedia, ACM Press, Santa Barbara.

[13] Achanta, R., Hemami, S., Estrada, F. and Süsstrunk, S. (2009) Frequency-tuned salient region detection. IEEE International Conference on Computer Vision and Pattern Recognition (CVPR), Miami.

[14] Sheikh, H.R. and Bovik, A.C. (2006) Image information and visual quality. IEEE Transactions on Image Processing, 15(2), 430-444.

[15] Sheikh, H.R., Wang, Z., Cormack, L. and Bovik, A.C. (2005) Live Image Quality Assessment Database Release 2. http:// live.ece.utexas.edu/research/quality.

[16] Mendi, E. and Milanova, M. (2010) Image quality assessment based on salient region detection. Journal of Visual Communication and Image Representation, Elsevier Ltd., 2010. 